\title{
Electrical Treeing Characteristics in XLPE Power Cable Insulation in Frequency Range between 20 and $500 \mathrm{~Hz}$
}

\author{
G. Chen \\ School of Electronics and Computer Science \\ University of Southampton, Southampton SO17 1BJ, UK
}

and C. H. Tham

SP Powergrid Ltd, Singapore

\begin{abstract}
Electrical treeing is one of the main reasons for long term degradation of polymeric materials used in high voltage ac applications. In this paper we report on an investigation of electrical tree growth characteristics in XLPE samples from a commercial XLPE power cable. Electrical trees have been grown over a frequency range from $20 \mathrm{~Hz}$ to $500 \mathrm{~Hz}$ and images of trees were taken using CCD camera without interrupting the application of voltage. The fractal dimension of electric tree is obtained using a simple box-counting technique. Contrary to our expectation it has been found that the fractal dimension prior to the breakdown shows no significant change when frequency of the applied voltage increases. Instead, the frequency accelerates tree growth rate and reduces the time to breakdown. A new approach for investigating the frequency effect on trees has been devised. In addition to looking into the fractal analysis of tree as a whole, regions of growth are being sectioned to reveal differences in terms of growth rate, accumulated damage and fractal dimension.
\end{abstract}

Index Terms - Electrical tree, fractal dimension, box-counting, variable frequency, growth rate, accumulated damage, partial discharge

\section{INTRODUCTION}

NOWADAYS, XLPE cables are widely chosen for power distribution and transmission lines up to $500 \mathrm{kV}$ owing to its excellent electrical, mechanical and thermal characteristics. Similar to any other insulating materials, its electrical properties deteriorate over the time when it is subjected to electrical stress. Electrical tree is one of the main reasons for long-term degradation of polymeric materials used in high voltage ac applications. Consequently, there have been continuous efforts in last three decades to characterize electrical treeing in XLPE and understand the mechanisms.

Electrical trees in solid insulation were firstly reported by Mason [1]. Subsequent research reveals that treeing is observed to originate at points where impurities, gas voids, mechanical defects, or conducting projections cause excessive electrical field stress within small regions of the dielectric. The treeing process can be generally described by the three stages, inception, propagation and runaway as shown in Figure 1. However, the exact form may vary depending on the mechanisms in operation.

Manuscript received on 15 January 2008, in final form on 14 November 2008.

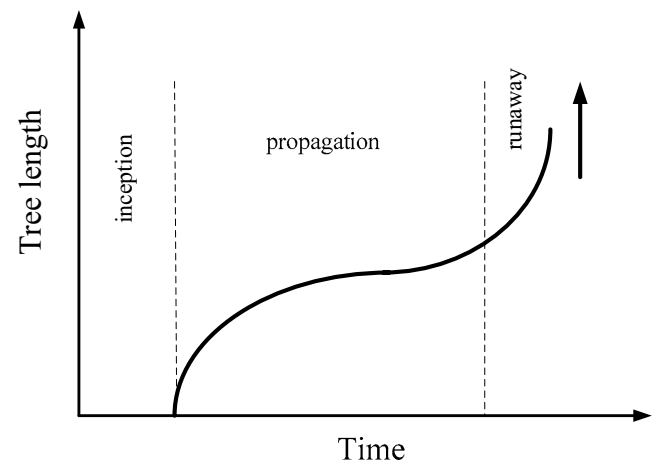

Figure 1. Electrical treeing growing characteristics.

One of the detailed early studies on electrical tree was carried out by Ieda and Nawata [2]. A few aspects were examined and the experimental results concluded that tree extension was induced by internal gas discharge in existing tree channel. The gas discharge was pulsive, lasting less than $0.1 \mu \mathrm{s}$ and the electric potential of a needle electrode was transferred to the tip of an existing tree channel through the conductive plasma of a gas discharge. It was also suggested that frequency only accelerates the growth process by increasing the number of gas discharges but not the nature of each discharge while the magnitude of local electric field at the tip of discharge columns was determined by the applied 
voltage. Noto and Yoshimura [3] examined polyethylene under various frequencies of ac electric stress. It was found that tree does not follow a linear growth relationship with the frequency. Under various applied voltages, tree exhibits different growth characteristics with various frequencies. The process of tree initiation decreased with increasing voltage and frequency and was assumed to be due to the increase in local electric field at the tip of the discharge column. Densley [4] studied the effects of frequency, voltage, temperature and mechanical stress on the time-to-breakdown (TTB) of XLPE cable insulation subjected to highly divergent field. It was found that trees grow in different shapes and colors at various frequencies and voltages. Tree shape changes at higher frequency which in turn reduces the TTB. TTB are also reduced significantly at higher temperatures and mechanical stress. The measurement of partial discharges (PD) also suggested the role of space charges were dominant in determining TTB and the shape of the tree. A comprehensive review of the developments made in the understanding of tree mechanisms was attempted by Dissado [5].

The concept of fractal dimension was firstly introduced to describe the geometrical characteristics of gas discharges by Niemeyer et al [6]. A simple two-dimensional stochastic model producing structures similar to those observed in experimental studies of branching gas discharges was established. Barclay et al [7] later constructed a twodimensional stochastic model of electrical treeing using a statistical method and from which they performed fractal analyses using a range of methods. It was found that trees of low fractal dimension, $D$ are the most dangerous as they grew faster across the pin plane space while trees of high fractal dimension grew slower but caused great amount of damages. Factors which reduce the fractal dimension increases the risk to the system. These include a crossover from bush-type to branch-type trees with higher voltage [4]. Smaller pin-plane spacing was also found to increase the branch density of trees formed [4], indicating that fractal dimension is determined by the local electric field, which will depend on both the applied voltage and the pin-plane spacing. Cooper and Stevens [8] studied the relationship between the fractal dimension of trees in a polyester and its bulk properties for various degrees of cross-linking. It was observed that the post-curing temperature of the resin influences the treeing behavior and the fractal dimension increases with increasing post-curing temperature and degree of cross-linking. Maruyama et al [9] revealed that a higher fractal dimension was resulted from a higher gel content. In the same study, the relation between the tree length and the fractal dimension was also made. It was observed that trees changes from branch-type to dense bush-type with increasing applied voltage and the fractal dimension increases with a stressing voltage of approximately $16 \mathrm{kV}$, after which it tends to saturate regardless of the increase in tree length. Fuji et al [10] examined the effect of the polarity of applied dc voltage on tree patterns obtained in polymethylmethacrylate (PMMA) samples. The studies pointed out that the fractal dimensions obtained at the two polarities differ and the fractal behavior depends on the local field or the space charge. A better understanding of fractal analysis and dimension applied on tree with various methods, both experimental and computational was done by Kudo [11]. The work estimated the fractal dimension of tree using methods such as boxcounting, fractal measure relations, correlation function, distribution function and power spectrum. It was found that there is a difference in fractal dimension obtained by the different methods and is unclear of the best method for estimating tree patterns. 3-D fractal analysis of real electrical trees had also been developed. It has been revealed in our recent research [12] that a double structure of electrical tree occurs when it grows at a submicroscopic structurally uneven region of the material. A new parameter, the expansion coefficient, was introduced to describe the electrical tree propagation characteristics.

Several models have been developed to simulate electrical tree growth. Stochastic model is popular due to its ease of computation [7]. The model is based on diffusion limited aggregation [13]. Simulated tree displays a remarkably similar behavior to experiment [7]. To understand the mechanism of electrical tree growth, physical process has been introduced to the stochastic model [14]. The addition of physical process helps to reproduce many of known characteristics of tree growth [15]. A deterministic treeing model has been proposed [16] and used successfully by Dodd [17] to study the growth of non-conductive electrical tree structures in epoxy resin. The model produces formation of branched structure of tree without the need of random variables. The more detailed description of these models can be found in literature [17]. Recently, cellular automata (CA) model has been reported to simulate tree growth [18]. Using a very simple rule, the tree formation was successfully reproduced in a dielectric with a point/plane electrode arrangement in the presence of voids. In addition to the above electrical models, the influence of mechanical stress on electrical tree growth has also been reported [19]. It has been demonstrated that the mechanical properties (specifically, tensile strength, elastic modulus and fracture toughness) of the dielectric material strongly affect the growth of electrical trees in single-cast homogeneous polyester resin specimens.

Despite these efforts, a full understanding has not yet been achieved due to complexity and various factors that may affect tree initiation and growth. In this paper we intend to investigate the influence of frequency on fractal dimension of electrical trees in XLPE under a fixed applied voltage. Methods for investigating the frequency effect on trees have been devised. Besides looking into the fractal analysis of tree as a whole, regions of growth are being sectioned to bring the study further.

\section{EXPERIMENTS}

\subsection{SAMPLE AND EXPERIMENTAL SETUP}

Semiconducting layer and conductor of a commercial XLPE cable, having an insulation thickness of $15 \mathrm{~mm}$, were removed, leaving only the insulation. Each cable specimen measuring $5 \mathrm{~mm}$ in lengths was then cut. The steel needle with a tip radius of $5 \mu \mathrm{m}$ was inserted gradually into the specimen to give a tip to earth-plane electrode separation of 
$2 \mathrm{~mm} \pm 0.2 \mathrm{~mm}$ at elevated temperature of between $120-140$ ${ }^{\circ} \mathrm{C}$. The sample was then annealed for approximately 5 minutes to minimize any mechanical stress build up around the pin-plane region before it was cooled down to room temperature. A typical sample with an inserted needle is schematically shown in Figure 2. All samples were inspected for the presence of mechanical stress around the pin tip region under polarized light. The samples with the presence of mechanical stress were discarded. Detailed information of preparing samples can be found in our research publications [12].

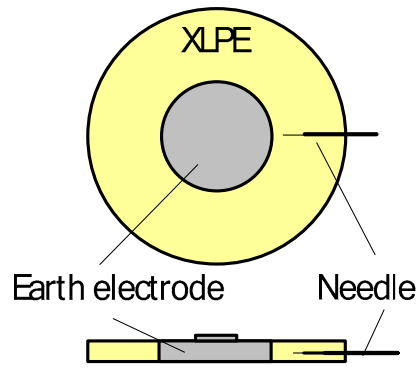

Figure 2. A schematic diagram of XLPE sample for treeing experiment.

The needle-plane specimen was kept in silicone oil cell to control the temperature and to prevent external discharges or flashover. They were subjected to continuous $7 \mathrm{kV}$ rms ac electrical stress over a range of frequencies. An average of six samples were tested at each frequency with testing frequencies at 20,50, 100, 300 and $500 \mathrm{~Hz}$. Prior to monitoring the growth of tree, all samples were pre-initiated using a $2 \mathrm{kHz}, 7 \mathrm{kV}$ AC voltage until a small $(50-80 \mu \mathrm{m})$ tree has formed at the tip. All the experiments were conducted at room temperature $\left(\sim 20^{\circ} \mathrm{C}\right)$. Densley [4] considered that once tree has initiated, it would have little or no effect on the subsequent growth of tree. Therefore, the pre-initiation will have limited effect on the result. A CCD camera (JVC TK1380) which is of sufficient spatial resolution to measure the spatial distribution of tree channel was then used to monitor electrical treeing optically during stressing. The skeletal structure of the tree was monitored by back lighting the sample with a projection lamp.

Images of evolving tree structures were captured periodically until the tree spanned approximately $90 \%$ of the pin-plane spacing. At this point, the test was terminated to protect both the external circuitry and the tree from damage in the event of a breakdown. The optical bench microscope was adjusted to a standard magnification level during all stages of tree growth so as to minimize errors due to the influence of magnification. The captured image was processed on the KS400 system developed by Imaging Associates Ltd. The experimental setup for treeing tests is shown in Figure 3. After that, the fractal dimension was computed with box-counting method.

\subsection{CALCULATION OF FRACTAL DIMENSION} 2.2.1 Image Acquisition and Segmentation

A high quality original image is an essential condition for accurate data analysis. The digitised image can be presented as binary, skeletonized or border-only image depending on the fractal dimension method used. It must also allow a clear distinction between the tree and the background, either by greyscale or by colors; to allow a simple thresholding operation. Thresholding provides an easy and convenient way to perform simple segmentation, an operation transforming digitized image into binary image required for data extraction; on the basis of the different intensities or colors in the foreground and background regions of an image. In the simplest implementation, the output is a binary image representing the segmentation. By looking at the image intensity histogram, the appropriate segmentation technique can be determined.

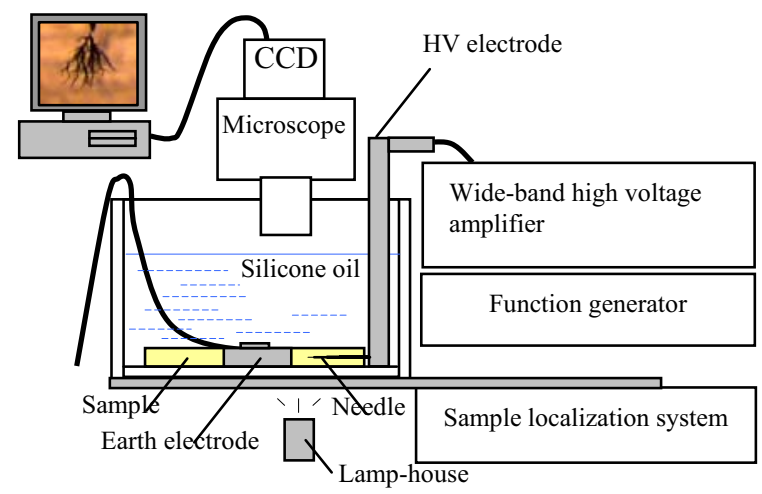

Figure 3. Experimental setup for treeing tests.

\subsubsection{Image Analysis and Measurement of Fractal Dimensions}

For automatic image analysis, the software-based imaging system KS400 was used. KS400 allows the development of application-specific macros which enables one to include all necessary functions in a single given application, i.e. image acquisition, calibration, processing, measurement and data output.

\subsubsection{Box Counting Method}

To estimate the box-counting fractal dimension, $D_{b}$, the 2-D Euclidean space containing the tree image was divided into a grid of boxes of size $\varepsilon$, with the initial box size being 1.3 times of the tree. Box size $\varepsilon$ was then made progressively smaller and the corresponding number of boxes, $N$, covering any part of the tree was counted. The sequence of box sizes for grids was usually reduced by a factor of half from one grid to the next. The count depends on box size $\varepsilon$ and $D_{b}$ according to eqnuation (1),

$$
N(\varepsilon) \propto \varepsilon^{D_{b}}
$$

Thus for a fractal structure a plot of $\log (\mathrm{N}(\varepsilon))$ against $\log (\varepsilon)$ should yield a straight line whose gradient corresponds to $D_{b}$,

$$
\log N(\varepsilon)=D_{b} \log (\varepsilon)+c
$$

where $c$ is a constant. 


\subsubsection{Data Processing}

The fractal dimension, $D_{b}$, may vary depending how it is obtained from the log-log plot. In such a plot, $D_{b}$ is related to the slope of the line, the number of data points being related to the number of measuring steps. The actual data points generally do not all lie on a straight line, thus showing limited self-similarity or scale invariance which is a characteristic of natural fractal object. Measured fractal dimensions can only be compared if this influence is excluded either by specifying a lower and upper limit for the linear regression or by introducing other criteria, such as defining a level of confidence in the R-squared value. It is an indicator from 0 (worst) to 1 (best) that reveals how closely the estimated values for the trend line correspond to the actual data. By applying the limits, certain data points were selectively rejected as long as the linear regression of the remaining data improved the R-squared value. In the present study, a program written as a macro for the KS400 system, has been used to obtain fractal dimension automatically.

\section{EXPERIMENTAL RESULTS}

\subsection{GROWTH RATE AND ACCUMULATED DAMAGE}

Two methods were used to analyze tree growth from a sequence of images captured periodically as tree grew from the tip to the earth-plane electrode. In the first method, arc of radii from an origin at the tip was drawn and the maximum tree extent from the tip was measured. The differences in tree length and time between photographs were used to compute a growth rate:

$$
\text { Growth } \operatorname{rate}(\mathrm{mm} / \mathrm{min})=\frac{\text { Max. Extent } 2-\text { Max. Extent } 1}{\text { Time } 2-\text { Time } 1}
$$

The other method is to measure the effective accumulated damages (area covered by tree structure in pixels) computed using the KS400 after image processing to obtain suitable binary images of tree structure. The area was estimated by extracting the total number of pixels covering the tree. Each pixel was to have an area of $4.255 \mu \mathrm{m} \times 4.255 \mu \mathrm{m}$. The two methods can effectively describe the spatial and temporal development of tree growth.

Images of the trees for various frequencies taken prior to breakdown are shown in Figure 4. They were stressed continuously at $7 \mathrm{kV} \mathrm{rms}$ and images were captured during tree growth and each image was separately analyzed to estimate the various parameters such as growth rate and accumulated damage. Variation in tree growth rate did exist within six samples tested for each frequency and data are only presented from reproducible trees. The selected results from $20 \mathrm{~Hz}, 50 \mathrm{~Hz}$ and $500 \mathrm{~Hz}$ are reported here to limit the length of the paper as the results from $100 \mathrm{~Hz}$ and $300 \mathrm{~Hz}$ are similar to those from $50 \mathrm{~Hz}$ and $500 \mathrm{~Hz}$, respectively.
A radial zone method [20] has been used to analyze tree growth at different frequencies. Figure 5 illustrates the growth rate and accumulated damage versus the pin-plane distance at $20 \mathrm{~Hz}$.

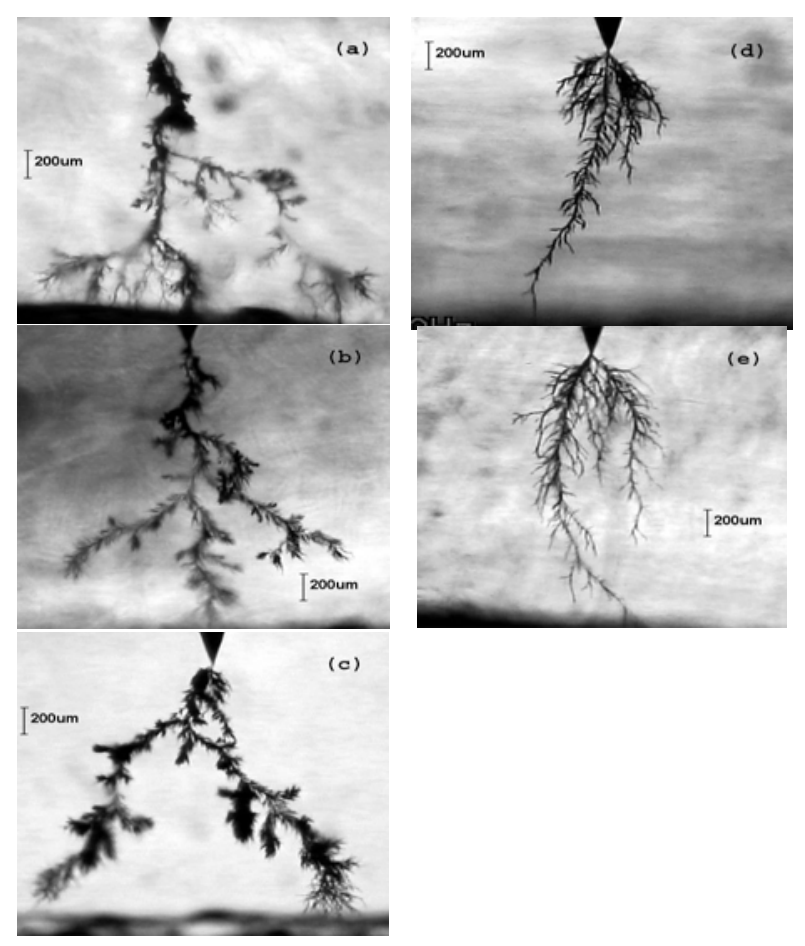

Figure 4. Captured photographs of tree growth for the XLPE cable samples prior to breakdown (a) $20 \mathrm{~Hz}$, (b) $50 \mathrm{~Hz}$, (c) $100 \mathrm{~Hz}$, (d) $300 \mathrm{~Hz}$ and (e) 500 $\mathrm{Hz}$.

It can be seen that during the initial growth stage, the tree displayed some rapid growth up to some $300 \mu \mathrm{m}$ from the pin tip in 10 minutes. It exhibited a high growth rate during that period but slowed down as tree extended away from the tip. Branching was concentrated (highly branched), extending to some $500 \mu \mathrm{m}$ and discernible damages were seen. The growth rate has dropped significantly as growth continued. Slow growth continues to be observed until the tree had advanced past $50 \%$ of the pin-plane distance followed by an increase in growth rate. At around $1200 \mu \mathrm{m}$, growth rate increased further and multiple branches were formed. It can be seen from the increase in accumulated damage. This latter region has often been identified as 'runaway' growth by many authors.

The accumulated damage (number of pixels) versus the tree length across the pin-plane spacing shows a general increase. Together with the growth rate, it suggests that tree actually exhibits three distinct growth regions. In region A, initial rapid filamentary tree growth occurs. Here the rate of damage increases with distance, with multiple branches being formed. Dense branching may also occur near the pin tip. This is followed by an intermediate region $\mathrm{B}$, in which low growth rate is observed. Finally, a region C occurs (the 'runaway' region), in which a large amount of damage per unit radial extent reflects the large increase in tree branching 
that occurs. In this region, it is also observed that the growth rate increases as leading branches extend towards the earth plane.

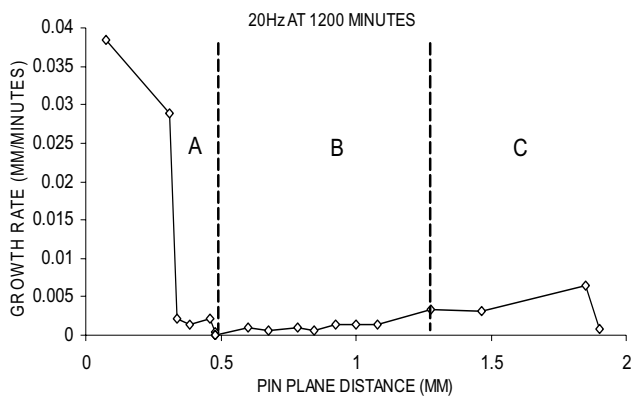

(a)

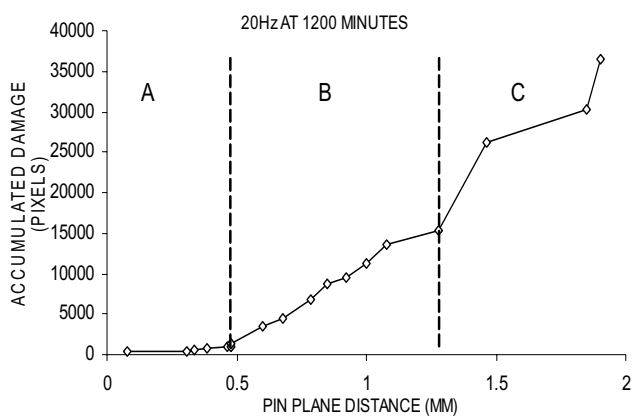

(b)

Figure 5. Growth rate (a) and accumulated damage (b) as a function of pinplane distance showing the three regions $\mathrm{A}, \mathrm{B}$ and $\mathrm{C}$ of tree growth.

Figure 6 illustrates the growth rate and accumulated damage versus the pin-plane distance at $50 \mathrm{~Hz}$. Samples stressed continuously with frequency at $50 \mathrm{~Hz}$ revealed that tree shape remains branch-type without significant changes to its shape. The tree exhibited a very rapid growth, extending up to some $500 \mu \mathrm{m}$ in the first 12 minutes. It can be seen from the high growth rate occurring in this region. After this initial growth, there was a long period of quiescence where there was very little or no significant growth from the leading branches. Accumulated damage versus the distance shows constant tree damage with length. During this period, partial discharge activities were known to be pre-dominant within the side-branches and channels which were lengthened and thickened in size. This observation can be further reinforced from Figure 6 that the tree had stopped extending but the number of pixels actually shown an increment in value. Subsequently, tree growth accelerated again after it had spanned past the $50 \%$ spacing.

Tree formed near the origin with a rapid growth was observed in the first 5 minutes at $500 \mathrm{~Hz}$ test as shown in Figure 7. There was a lessening in growth after initial activity with growth rate comparable to those observed at low frequency. Large accumulated damage occurred near the origin followed by one or two leading branches extending outward till breakdown. Tree growth can be split into two regions with A-C transition occurs at $1400 \pm 100 \mu \mathrm{m}$. In region $\mathrm{C}$, the leading branch takes less than 10 minutes to breakdown, influenced by the high growth rate.

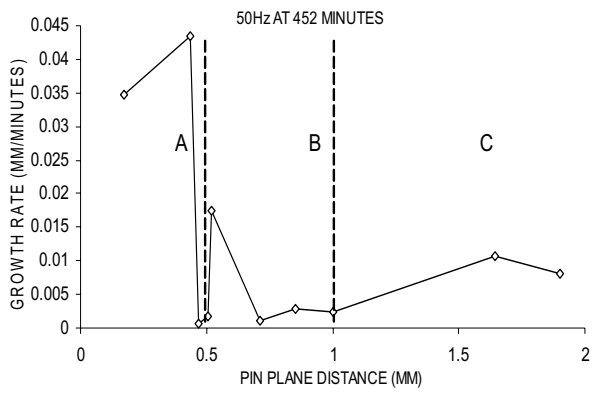

(a)

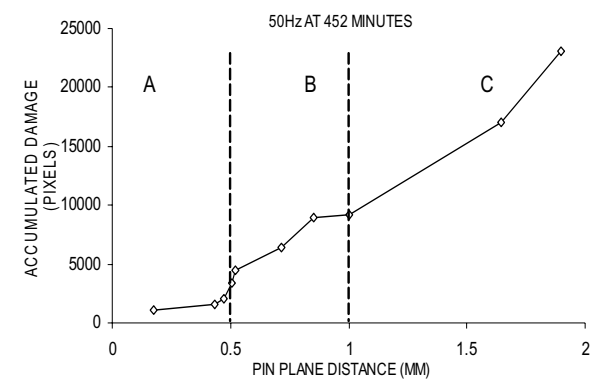

Figure 6. Growth rate (a) and accumulated damage (b) as a function of pinplane distance showing the three regions $\mathrm{A}, \mathrm{B}$ and $\mathrm{C}$ of tree growth.

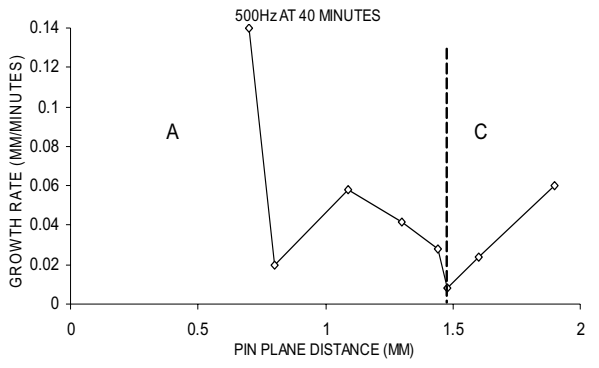

(a)

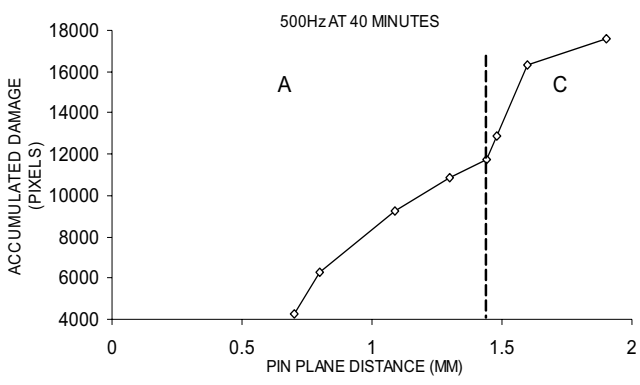

(b)

Figure 7. Growth rate (a) and accumulated damage (b) as a function of pinplane distance showing the two regions $\mathrm{A}$ and $\mathrm{C}$ of tree growth.

\section{DISCUSSIONS}

\subsection{Growth Characteristics of Electrical Tree}

While the success of the growth rate and accumulated damage methods may have provided the study of tree growth into regions, the methods are not easily incorporated into other models found in the literature such as the field driven tree growth (FDTG) model [20]. Growth rate computed by taking the maximum radial extent of the tree is found that the 
leading branch taken as the largest extent may stop growing for some time while other side branches from the origin continue to grow. This method may be ineffective in taking account of growth occurring at other branches with shorter radial extent. Therefore, the accumulated damage method was devised to look into the spatial development of tree more accurately.

From analyses, tree exhibits two or more regions of growth and the fractal dimension in each region is dissimilar as shown in Table 1. Thus the variation in local field $E_{L}$ may have the effect of affecting the fractal dimension. It is believed there are some variances in $E_{L}$ instead of equaling in magnitude in both regions since the fractal dimensions are different, which is thought to be affected by $E_{L}$. The difference in growth duration at each region can further reinforce this analysis.

Table 1. Fractal dimension of tree growth in different regions.

\begin{tabular}{|c|c|c|c|}
\hline $\begin{array}{c}\text { Frequenc } \\
\text { y } \\
(\mathrm{Hz})\end{array}$ & Region A & Region B & Region C \\
\hline 20 & 1.79 & 1.71 & 1.70 \\
\hline 50 & 1.69 & 1.71 & 1.64 \\
\hline 100 & 1.70 & 1.63 & 1.66 \\
\hline 300 & 1.68 & - & 1.59 \\
\hline 500 & 1.66 & - & 1.54 \\
\hline
\end{tabular}

It is also noted that tree exhibits same type of growth characteristic with a high initial growth rate followed by a period of quiescence (for tree with two growth regions), then a 'runaway' region. The initial high growth may be attributed by the field enhancement near the pin tip. With tree approaching the earth-plane electrode, field is once again enhanced. Other explanations may be given for this behavior such as image charges intensifying the local field on approaching the plane electrode [14].

Analysis of six tested samples indicates some variation in the positions of boundary between different growth regions. The results shown in Figures 5-7 were taken from those samples with positions sitting approximately in the middle. The actual positions of the boundary can be affected by several factors, including morphological features in different samples and variation in local electric field during tree growth.

\subsection{FREQUENCY-DEPENDENT TREE BREAKDOWN}

Trees shown in Figures $4 \mathrm{a}$ to $4 \mathrm{c}$ are often known as 'monkey puzzle' (MP) tree [14]. It is in the last two regions that tree takes on the appearance of a MP tree. This type of structure is an open structure on the macroscopic level with microscopic secondary branching existing along the length of each main tree channel, the branch structure having a hairy spider's leg appearance. It is known to grow with stressing voltage at low voltage [19]. At increasingly higher stressing frequency, MP tree is not featured as branch density dropped.

In Figure 8, it shows that tree radial extent follows quite a linear relationship with frequency at the early stage. At higher frequency, tree extends faster through fine filamentary branches. This type of behavior has been reported to occur [3]. Figure 9 shows TTB as a function of the stressing frequency. TTB is seen to be faster at higher frequency and comparing the accumulated damage at different frequencies, it shows that TTB decreases with lower accumulated damage. Therefore, TTB also depends on the distribution of accumulated damage.

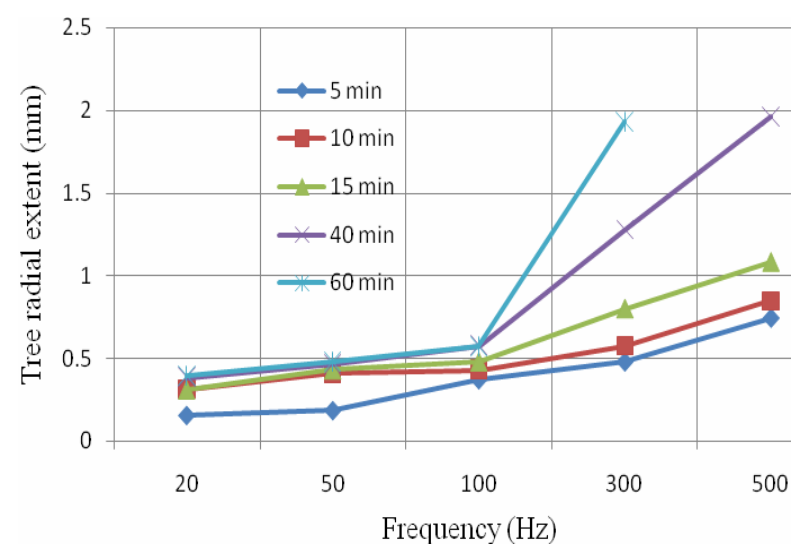

Figure 8. Tree radial extents as a function of frequency showing tree length at various times.

\subsection{FRACTAL DIMENSION--- REGIONS A, B AND C}

The fractal nature of tree is demonstrated by the consistency of the fractal dimension estimates obtained. Disregarding the very small trees grew initially; value of 1.55 to 1.71 is always obtained for five frequencies tested without a clear trend. It tends to saturate in this range as trees were of branch-type. Thus, there is no significant change to the fractal dimension. This branch-type characteristic has been shown to produce under a stressing voltage of $7 \mathrm{kV}$ [3]. Kudo [11] performed a fractal analysis on the photographs of the branch trees (Figure 9 of [3]), which were stressed at $7 \mathrm{kV}$, has an estimated fractal dimension of around 1.6. This value coincides closely with data here.

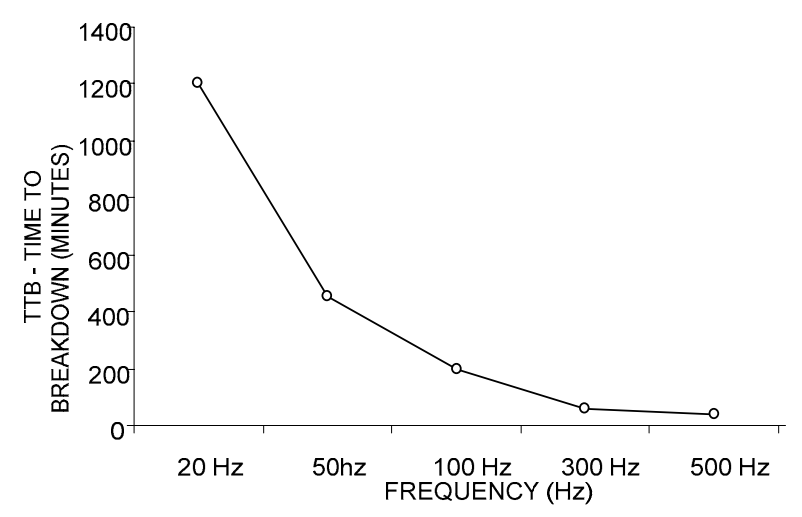

Figure 9. The time to breakdown against frequency.

As fractal dimension for different frequencies varies randomly from 1.55 to 1.71 , it is difficult to draw any inference. On the other hand, there are different stages during tree growth, it would be interesting to look at the fractal 
dimension in each region. Images were sectioned into three regions using the approximated transition zone and the fractal dimension of each region was computed using the KS400 system as shown in Table 1.

It can be seen that the value of fractal dimension in region $\mathrm{A}$ is always higher than $\mathrm{C}$ for all the frequencies. In region $\mathrm{C}$, tree only constitutes of a few leading branches to form the complete breakdown path, resulting in a lower fractal dimension than in region A. At frequency of above $300 \mathrm{~Hz}$, fewer branches were formed, leading to a lower fractal dimension than at lower frequency. Dissado et al [14] show that a bush tree with higher fractal dimension developed from higher voltage $(\sim 12-15 \mathrm{kV})$ takes a longer time to breakdown than branch tree with lower fractal dimension at lower voltage $(\sim 9-11 \mathrm{kV})$. The damage in region $A$ at lower frequency is more concentrated than at higher frequency. Fine filamentary branches having a larger radial extent from the tip were seen at higher frequency in region $\mathrm{A}$, thus resulting in lower fractal dimension.

Using the knowledge of higher fractal dimension resulting in longer breakdown from Dissado's work, it appears tree growth in region A may actually control the TTB. Thus, a regression line describing the empirical relationship relating the fractal dimension and TTB is shown in Figure 10. It may be possible to estimate the TTB by estimating the fractal dimension of the growing tree in which the fractal dimension in region A may be the controlling factor for TTB.

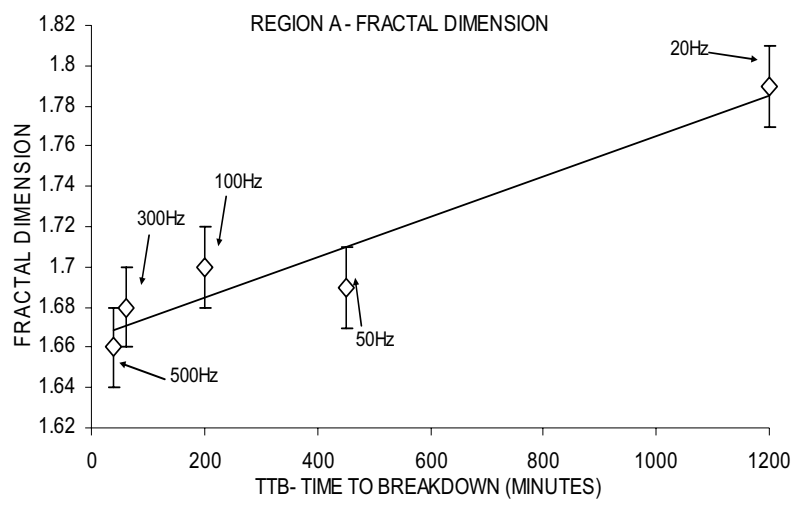

Figure 10. Correlation between the fractal dimension of region A and TTB.

\subsection{ACCUMULATED DAMAGE - REGIONS A, B AND C}

The devised accumulated damage method was also applied to the different regions similar to that of fractal analysis and the results are shown in Table 2 .

More accumulated damages appear to have been done to region $\mathrm{A}$ at higher frequency. This is due to a larger region exhibited from the tests and a higher branching density near the tip. In the same region, the branch density is higher with finer filamentary branches spreading at a wider arc angle from the tip. At lower frequency, thicker branches were densely packed together resulting in a solidly filled structure and the branching is concentrated with only one or two such branches present.
Table 2. Number of pixels accumulated in different growth regions.

\begin{tabular}{|c|c|c|c|}
\hline $\begin{array}{c}\text { Frequenc } \\
\text { y } \\
(\mathrm{Hz})\end{array}$ & Region A & Region B & Region C \\
\hline 20 & 3303 & 11230 & 23864 \\
\hline 50 & 3625 & 6930 & 16925 \\
\hline 100 & 5126 & 5148 & 13331 \\
\hline 300 & 11842 & - & 2073 \\
\hline 500 & 17539 & - & 1797 \\
\hline
\end{tabular}

In region $\mathrm{C}$, only a few runaway branches leading to breakdown at higher frequency are formed, giving lower damages. Tree growth occurs at all tree tips at low frequency lead to tree structures in these region having a higher branching density than in region $\mathrm{B}$, resulting in higher damages made. Like the regression line describing the empirical relationship relating the fractal dimension and TTB shown in Figure 10, a similar regression line in Figure 11 is made to illustrate the possibility of calculating the TTB by estimating the accumulated damage of the growing tree in which the accumulated damage in region $\mathrm{C}$ may be the controlling factor for TTB.

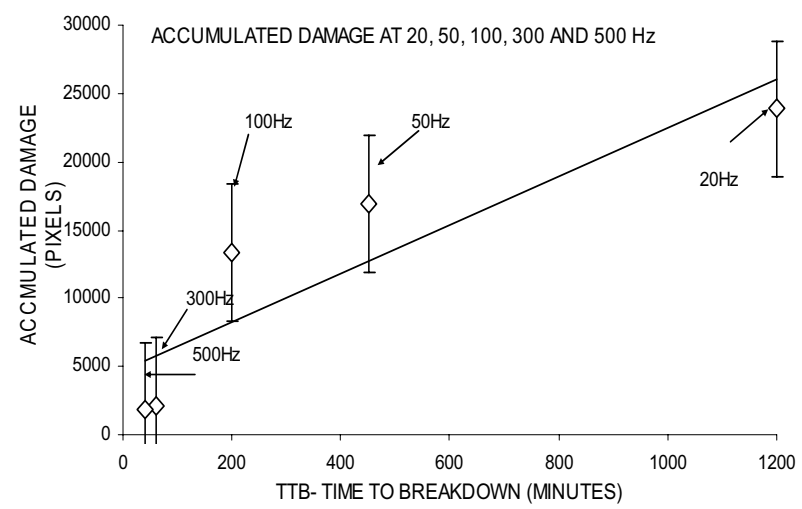

Figure 11. Regression line describing the empirical relationship relating the damage and TTB in region $\mathrm{C}$.

\subsection{NUMBER OF CYCLES}

Partial discharges (PD) are typical measured and studied at an operating frequency of the applied voltage, in general $50 / 60 \mathrm{~Hz}$. However, there are many studies measuring PD at other applied frequencies. A lower applied frequency reduces the power and size needed for the voltage supply equipment. Alternatively PD can also be studied at variable applied frequencies to provide more information than measurements at a single frequency as the variation in applied frequency changes the local state at defects in the insulation [21]. It has been stated that these changes can be used to better characterize the defects, provided that the frequency dependence of PD is interpreted physically.

An empirical relationship relating the number of cycles experienced by the tree and the time to breakdown is established by the regression line shown in Figure 12.

The relationship is determined using,

$$
\text { Numberof Cycles }=\frac{\text { TTB } \times 60 \text { seconds }}{\text { Time Taken per Cycle }}
$$




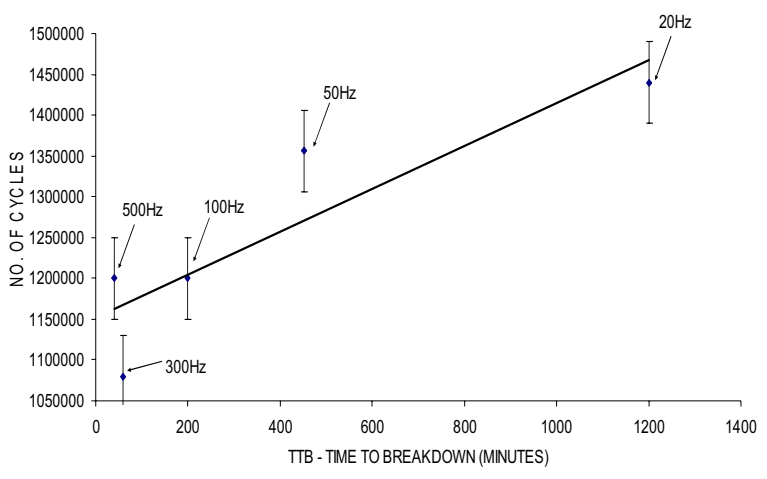

Figure 12. The number of cycles experienced by tree before breakdown.

It shows that tree does not experience the same number of cycles before its breakdown. This may be due to the time taken for charges to transport through tree branches which are not affected by frequency. Other factors such as morphology effect may play a role in the breakdown. Using the same figure, it may also show the invalidity of trying to correlate the data to partial discharge (PD) monitoring at frequencies other than $50 \mathrm{~Hz}$. From the treeing study, the data may correlate the relationship since tree extension is due to PD activities in the channels. It is found that the concept of monitoring PD in cable at lower frequency may not be fully consistent with that at power frequency. Though, the number of discharges occurring per cycle is lower at higher frequency, the total number of discharges is actually higher for a fixed period. Therefore, further experimental and theoretical works are required.

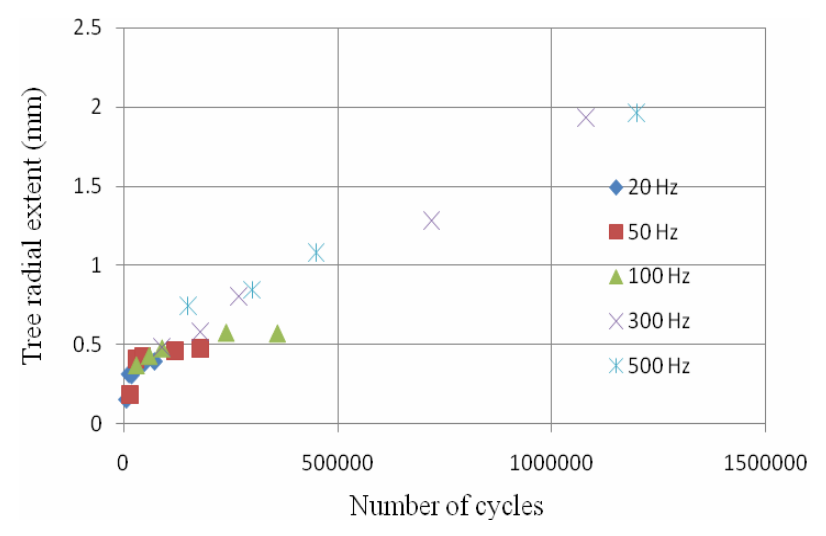

Figure 13. Electrical tree growth as a function of the number of cycles.

To further validate the relationship between the tree growth and number of field cycles, Figure 13 shows the tree radial extent against the number of cycles at various frequencies. It seems that the tree length increases linearly with the number of cycles experienced. This linear relationship may suggest that the mechanical fatigue is responsible for tree growth in XLPE. Analysis carried on fractal dimension in regions A and $\mathrm{C}$ as a function of the number of cycles also indicate that there is a general increase in fractal dimension in both regions. This further strengthens that the fatigue process is related to the tree growth.

\subsection{MATERIAL-DEPENDENT CRITICAL FIELD}

The description of the propagation curves may be able to provide some insight on tree growth affecting the fractal dimension and the simple field driven tree growth (FDTG) model [20] is being used and will be briefly described here.

The model is constructed by taking the assumption that the incremental increase in accumulated damage occurs during an ac stressing cycle is proportional to the magnitude of the local electric field $E_{L}$ exceeding a material-dependent critical field $E_{c}$. If the tree is conducting with the presence of PD activity, it will modify $E_{L}$ by effectively reducing the pinplane spacing and increasing the apparent pin-plane radius. As tree branches extend beyond the pin tip, $E_{L}$ can be related to the new tree length, $L$ by taking the tree to be effectively a conducting hyperboloid extension of the point electrode. This assumption gives the maximum field along the hyperboloid axis as,

$$
E_{L}=E_{M A X}=\frac{2 V}{(L+r) \ln \left(\frac{1+a}{1-a}\right)}
$$

where $r$ is the radius of curvature of the pin tip, $V$ is the peak value of the applied voltage, and

$$
a=1+\frac{L+r}{w-L}
$$

with $w$ the pin-plane distance. This expression is equivalent to that given by Mason [22]. From expression, $E$ is a function of $L$, thus the magnitude changes as tree grows. Initially $E$ will decrease as the equivalent hyperboloid radius increases, leading to decelerating growth. When $E_{L}$ drops below $E_{c}$ (indicated by an arbitrary line in Figure 14) the growth will slow to a stop. However, before this could happen, the field may start to increase again as the earth-plane electrode is approached. As a result tree growth will start to accelerate again. Such behavior is typical of many electrical trees [23].

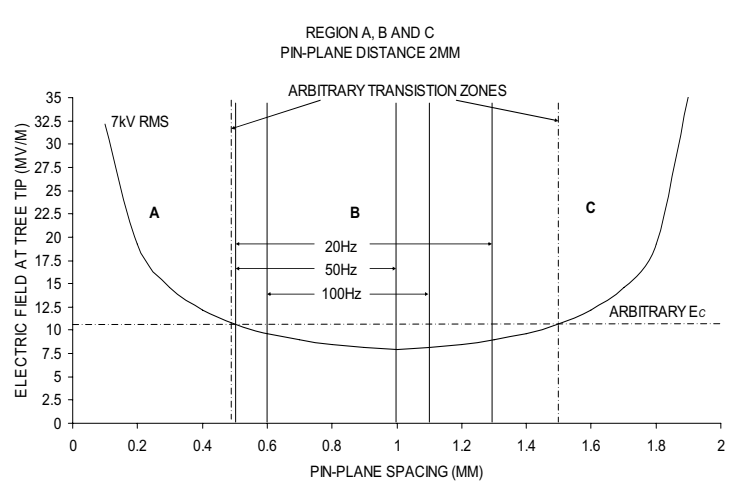

Figure 14. Local electric field at the damage perimeter as a function of damage extent for tree growth with 2 transition zone. Dotted line shows the ideal characteristic of the FDTG model.

Plots of $E_{L}$ against the hyperbolic radius using equation (5) is shown in Figures 14 and 15 under $7 \mathrm{kV}$ RMS with the pinplane distance being $2 \mathrm{~mm}$. Here, the curve shows how $E_{L}$ varies as the tree grows across the pin-plane gap. From the figures, it demonstrates the different regions present without a variation in $E_{L}$. Comparing it with the ideal transition zones 
from the model, it found limitations with the results. The model fails to infer $E_{c}$ graphically. In the literature, one transition zone was found when a higher value of $E_{L}$ was applied. Since equation (5) does not take into consideration of frequency when computing the local field, $E_{L}$ remains constant with varying frequencies. One of the assumptions in the FDTG model is that the tree can be considered as a conducting hyperboloid extension of the pin tip. From Table 1 , it is known that the fractal dimension of tree decreases with frequency, this indicates that the assumption may not hold for trees at high frequencies where a low fractal dimension of tree has been observed. On the other hand, the FDTG model assumes that there is no potential drop as the tree is considered forming from conducting branches. Again, this may not hold all the time, leading to an overestimation of $\mathrm{E}_{\mathrm{L}}$ in magnitude. This will affect the tree growth characteristics.

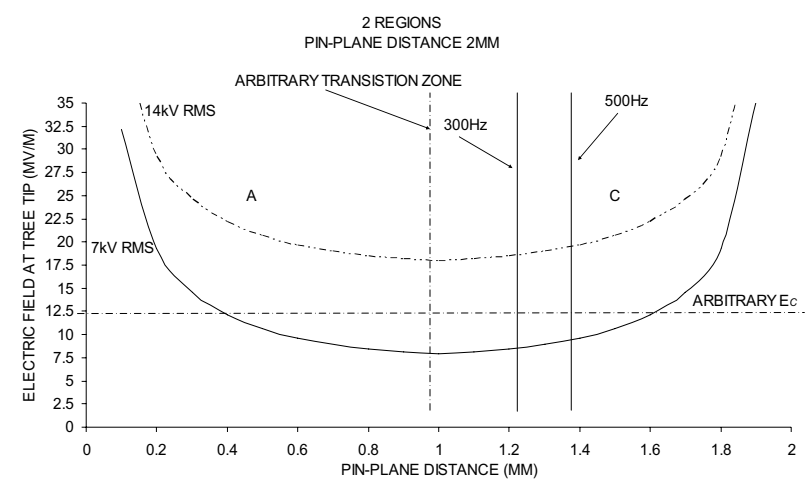

Figure 15. Local electric field at the damage perimeter as a function of damage extent for tree growth with one transition zone. Dotted line shows the ideal characteristic of the FDTG model when using the same sample, the field will not drop below $\mathrm{E}_{\mathrm{c}}$ with a higher stressing voltage.

In the literatures, two transition zones were found at low stressing voltage and one at high stressing voltage. Thus, it is interesting to see such similar behavior where there are also two transition zones at low frequency and one at high frequency. Therefore, the frequency has an effect of modifying the number of growth regions even though $E_{L}$ does not change. Despite the simplicity of the FDTG model, it has been successfully applied to spatial and temporal observations of electrical tree growth in synthetic resins [20].

\section{CONCLUSIONS}

Tree growth under the influence of frequency is not able to infer much with fractal dimension due to the similar branchtype structure formed. Instead, it is found to have accelerated the breakdown process with higher frequency leading to a faster breakdown. This could be due to the higher number of partial discharges at higher frequency. Fractal dimension is thus thought to be influenced primarily by the magnitude of stressing voltage as frequently discussed in the literature.

Further analyses were made and by using the devised approaches, tree growth can be sectioned into two or three regions depending on the frequency applied. At low frequency, three regions are present in the growth process while there are only two at higher frequency. The first is region A, where high growth rate is exhibited in which filamentary tree growth occurs at the pin tip. Next is region B in which tree growth is slowed and the fractal dimension is lower than in region A. Lastly in region $\mathrm{C}$, growth rate picks up with an increase in branch density and decrease in accumulated damage as tree extends towards the earth-plane electrode. Again, the fractal dimension is lower than in region A. Therefore, the regions analyses suggest that the time-to-breakdown is primarily influenced by the fractal dimension in region $\mathrm{A}$ and the amount of tree damage in region $\mathrm{C}$.

Tree is found not to have experienced the same number of cycles, thus partial discharges before its breakdown at various frequencies. It is thought that the time taken to transport charges from the origin to the tree tip may have affected the number of discharges occurring. It also shown that the correlation of partial discharge activity at lower frequency with power frequency through total number of cycles tree experiences is not explicit and does not follow a linear relationship. On the other hand, the tree growth shows a nearly linear relationship with the number of field cycles. This may suggest that the mechanical fatigue is responsible for tree growth in XLPE.

\section{REFERENCES}

[1] J. H. Mason, "The Deterioration and Breakdown of Dielectric Resulting From Internal Discharges", Proc. IEE, Vol. 98, pp. 44-59, 1951.

[2] M. Ieda and M. Nawata, "Consideration of Treeing in Polymers", IEEE Conf. Electr. Insul. Dielectr. Phenomena (CEIDP), pp. 143-150, 1972.

[3] F. Noto and N. Yoshimura, "Voltage and Frequency Dependence of Tree Growth in Polyethylene", IEEE Conf. Electr. Insul. Dielectr. Phenomena (CEIDP),, pp. 206-217, 1974.

[4] R. J. Densley, "An Investigation Into The Growth of Electrical Trees in XLPE Cable Insulation”, IEEE Trans. Electr. Insul, Vol. 14, pp.148$158,1979$.

[5] L. A. Dissado, "Understanding Electrical Trees in Solids: From Experimental to Theory", IEEE Trans. Dielectr. Electr. Insul, Vol. 9, pp. 483-497, 2002.

[6] L. Niemeyer, L. Pietronero and H.J. Wiesmann, "Fractal Dimension of Dielectric breakdown", Phys. Rev. Lett, Vol. 52, pp. 1033-1036, 1984.

[7] A. L. Barclay, P. J. Sweeney, L. A. Dissado and G. C. Steven, "Stochastic Modeling of Electrical Treeing: Fractal and Statistical Characteristics", J. Phys. D: Appl. Phys. Vol. 23, pp. 1536-1545, 1990.

[8] J. M. Cooper and G. C. Steven, "The influence of Physical Properties on Electrical Treeing in a Cross-Linked Synthetic Resin", J. Phys. D: Appl. Phys., Vol.23, pp. 1528-1535, 1990.

[9] S. Maruyama, S. Kobayashi and K. Kudo, "Fractal Characteristics of Real Electrical Trees", IEEE $4^{\text {th }}$ Int. Conf. Conduction \& Breakdown Solid Dielect, pp. 318-322, 1992.

[10] M. Fuji, M. Watanabe, I. Kitani and K. Yoshino, "Fractal Character of dc Trees in Polymethylmethacrylate", IEEE Trans. Electr. Insul., Vol. 26, pp. 1159-1162, 1991.

[11] K. Kudo, "Fractal Analysis of Electrical Trees", IEEE Trans. Dielectr. Electr. Insul,, Vol. 5, pp. 713-727, 1998.

[12] X. Zheng and G. Chen, "Propagation Mechanism of Electrical Tree in XLPE Cable Insulation by Investigating a Double Electrical Tree Structure", IEEE Trans. Dielectr. Electr. Insul, Vol. 15, pp. 800 - 807, 2008.

[13] H. J. Wiesmann and H. R. Zeller, "A fractal model of dielectric breakdown and prebreakdown in solid dielectrics", J. Appl. Phys. Vol. 60, pp. $1770-1773,1986$.

[14] L. A. Dissado, S. J. Dodd, J. V. Champion, P. I. Williams and J. M. Alison, "Propagation of electrical tree structures in solid polymeric insulation”, IEEE Trans. Dielectr. Electr. Insul,, Vol. 4, pp. 259 - 279, 1997. 
[15] K. Wu, Y. Suzuoki, T. Mizutani and H. K. Xie, "A novel physical model for partial discharge in narrow channels", IEEE Trans. Dielectr. Electr. Insul. Vol. 6, pp. 181 - 190, 1999.

[16] L. A. Dissado, J. C. Fothergill, N. Wise, A. Willby and J. Cooper, “A deterministic model for branched structures in the electrical breakdown of solid dielectrics", J. Phys. D: Appl. Phys. Vol. 33, pp. 99 - 112, 2000.

[17] S. J. Dodd, "A deterministic model for the growth of non-conducting electrical tree structures", J. Phys. D: Appl. Phys. Vol. 36, pp. $129-$ $141,2003$.

[18] M. Danikas, I. Karafyllidis, A. Thanailakis, and A. Bruning, "Simulation of electrical tree growth in solid dielectrics containing voids of arbitrary shape",. Modelling Simul. Mater. Sci. Eng., Vol. 4, pp. 535.552, 1996

[19] B. R. Varlow and D. W. Auckland, "The Influence of Mechanical Factors on Electrical Treeing”, IEEE Trans. Dielectr. Electr. Insul., Vol. 5, pp. 761-766, 1998

[20] J. V. Champions, S. J. Dodd, and G. C. Stevens, "Analysis and Modelling of Electrical Tree Growth in Synthetic Resins Over a Wide Range of Stressing Voltage", J Phys. D: Appl. Phys., Vol.27, pp. 10201030, 1994.
[21] H. Edin, Partial Discharges Studied with Variable Frequency of the Applied Voltage, Ph. D. Thesis, KTH, Stockholm, Sweden, 2001.

[22] J. H. Mason, "Breakdown of solid dielectrics in divergent fields", Proc. IEE., Vol.102, pp. 254-63, 1955.

[23] L. A. Dissado and J.C. Fothergill, Electrical Degradation and Breakdown in Polymers", IEE Materials and Devices Series 9, P. Peregrinus, London 1992.

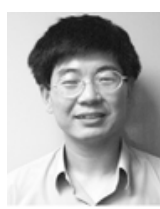

George Chen was born in China in 1961. He received the B.Eng. and M.Sc. in 1983 and 1986, respectively in electrical engineering from Xian Jiaotong University, China. After he obtained the Ph.D. degree in 1990 in electrical engineering from The University of Strathclyde, UK, he joined the University of Southampton as a postdoctoral research fellow and became a senior research fellow subsequently. In 1997 he was appointed as a research Lecturer and was promoted to a Reader in 2002. Over the years, he has developed a wide range of interests in high voltage engineering and electrical properties of materials and published over 150 papers. 